\title{
Recent Advancement of Slow Light in Microwave Photonics Applications
}

\author{
Sanghoon Chin and Luc Thévenaz \\ Ecole Polytechnique Fédérale de Lausanne, STI-GR-SCI-LT Station 11 \\ CH-1015 Lausanne, Switzerland \\ sanghoon.chin@epfl.ch
}

\begin{abstract}
A complete realization of an optically tunable true time delay, generated through the combination of a photonic $R F$ phase shifter and a Brillouin slow light element is presented. Illustration through a dynamic microwave photonic filter is demonstrated.
\end{abstract}

\section{INTRODUCTION}

The development of dynamic photonic delay lines is of major importance for broadband digital and analogue signal processing in communication systems. In particular, optical delay lines are extensively studied for microwave systems such as phased array antennas (PAA), microwave photonic filters (MPF) and arbitrary waveform generators, due to their inherent advantages such as large bandwidth, capability of operation over entire RF frequency with low loss and high delay time-signal bandwidth product [1]. Among the wide diversity of optical delay line schemes that have been reported over the last few years [2,3], slow and fast light (SFL) has been devised as one potential approach to generate continuously tunable signal delays. However, the apparent perfect true time delay (TTD) generated by slow light suffers from two major obstacles: the nearly finite delay time-signal bandwidth product and the strictly limited operating frequency. It means that only modest maximal time delay can be expected at high RF frequencies and the operating frequency is essentially limited by the bandwidth of the induced optical resonance, which prevents its complete implementations for applications in microwave photonics.

To overcome these limitations, a new technique, named separate carrier tuning (SCT), was recently proposed and theoretically investigated. This architecture predicts that significant improvement for the generation of true time delay can be achieved by separately performing photonic microwave phase shifting and true time delaying using slow light in micro-resonators [4]. Numerous radar systems have a low operating frequency (down to few $\mathrm{GHz}$ for ground-based radar) and therefore require up to $10 \mathrm{~ns}$ of TTD. It is known

We acknowledge the support from the Swiss National Science Foundation through project 200020-121860 and from the European Community's Seventh Framework Programme [FP7/2007-2013] under grant agreement $n^{\circ} 219299$ (GOSPEL project) that stimulated Brillouin scattering (SBS) gain resonance induces a dispersion which corresponds to delays up to few tens of ns, but over a limited bandwidth [5]. In this paper, we present a continuously tunable TTD line, in which both phase compensation and slow light delaying are only controlled by stimulated Brillouin scattering in optical fibers, upgrading a former version implemented to verify the validity of the SCT approach [6]. A complete analysis of the performances of this tunable TTD line for the development of phased array antenna systems and dynamic microwave photonic filters is presented. In addition, the high flexibility of the system, in terms of operating frequency and signal bandwidth, is discussed.

\section{PRINCIPLE}

Let us consider a single sideband modulated optical signal comprising an optical carrier at frequency $v_{c}$ and a modulation sideband at frequency $v_{c}+v_{R F}$. A first spectrally broadened Brillouin pump induces a broadband resonance centered at $v_{c}+v_{R F}$. The signal group delay is then given by the slope of the optical phase at $v_{c}+v_{R F}$, as depicted in Figure 1:

$$
T_{s}=\left.\frac{1}{2 \pi} \frac{\partial \phi}{\partial v}\right|_{v_{c}+v_{R F}},
$$

In broadband applications, the RF signal has a finite bandwidth $\Delta v_{R F}$ of typically $10-30 \%$ of the operating $\mathrm{RF}$ frequency $v_{R F}$. TTD operation implies a constant group delay over the entire frequency range including the carrier and the modulation sideband. Since no information is conveyed in the a major part of the spectral range between the optical carrier frequency and the modulation sideband centered on the RF signal, TTD operation is simply achieved by calculating the phase shift necessary to make signal and RF group delays match, deduced through the simple expression [4]:

$$
T_{R F}=\frac{\phi\left(v_{c}+v_{R F}\right)-\phi\left(v_{c}\right)}{2 \pi v_{R F}}=-\frac{\phi\left(v_{c}\right)}{2 \pi v_{R F}},
$$




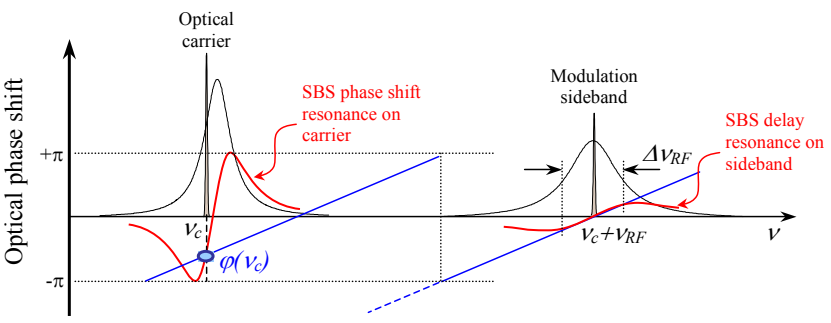

Figure 1: Principle to correctly operate true time delay over the RF signal bandwidth $\Delta v_{R F}$ simply by controlling dispersion properties, separately at carrier frequency and signal bandwidth. $v_{c}$; optical carrier frequency, $\varphi\left(v_{c}\right)$; optical phase shift introduced to the optical carrier, $v_{R F} ; \mathrm{RF}$ signal microwave frequency).

since the phase shift at the center of the Brillouin resonance $\varphi\left(v_{c}+v_{R F}\right)$ is zero. This situation can be effectively realized by separately managing the dispersion properties at the carrier frequency and around the signal bandwidth, as shown in Figure 1.

In our system, a second Brillouin pump generates a narrow-band resonance in the vicinity of the carrier frequency. The phase of the optical carrier is then adjusted to ensure $T_{R F}=T_{s}$, by simply detuning the central frequency of the resonance. As a result, it makes possible to generate correct true time delay for the whole signal bandwidth while dispersion across the entire bandwidth is no longer required unlike typical slow light systems. Also, it must be pointed out that since this optical phase shift is imparted on the carrier, the corresponding phase shift of the electrical signal is therefore constant with respect to the RF frequency. As a consequence, the adjustment of the optical carrier phase $\varphi\left(v_{c}\right)$ can be performed modulus $2 \pi$. A $360^{\circ}$ tunability of the optical carrier phase is thus sufficient to achieve TTD operation. Within the RF bandwidth of interest, this type of TTD generation is conceptually similar to a slow light-based TTD with a dispersion induced by a single broadband resonance centered on $v_{c}$, with FWHM bandwidth of $2 v_{R F}$. Yet, this new architecture shows two crucial advantages: first, it is independent of the operating RF frequency since the optical resonance can be flexibly displaced at any desired RF frequency; second, it can produce a large TTD with a proper bandwidth at high frequency RF signals since the signal bandwidth of interest is substantially lower than the RF signal frequency.

\section{EXPERIMENTAL LAYOUT}

The schematic diagram of our experimental setup is depicted in Figure 2, in which two distinct Brillouin slow light systems are basically combined. A 20-km-long dispersion shifted fiber (DSF) was used as a Brillouin gain medium and the Brillouin characteristics of this fiber were measured, showing a Brillouin shift $v_{B}$ of $10.73 \mathrm{GHz}$ and an SBS gain bandwidth $\Delta v_{B}$ of $27 \mathrm{MHz}$. A commercial distributed feedback (DFB) laser diode operating at $1551 \mathrm{~nm}$ was utilized as a light source to generate the optical carrier for the microwave signal and the two Brillouin pumps using a set of wideband electro-optic modulators (EOM). This way we can obtain a high stability of the frequency difference between the signal and the two

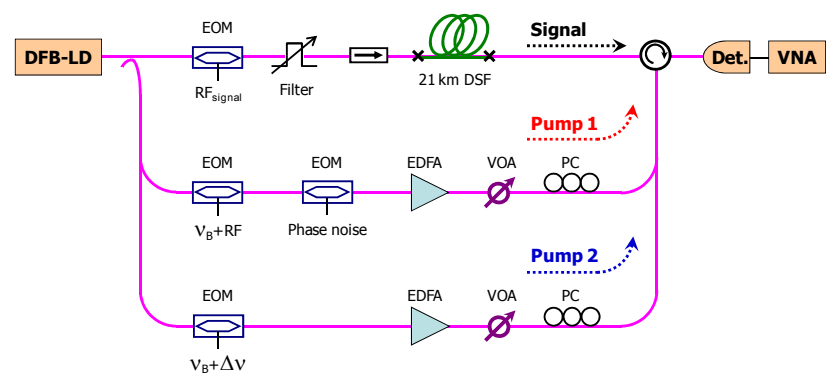

Figure 2: Schematic diagram of the generation of tunable true time delay, based on separate carrier tuning method.

Brillouin pumps [7]. The output light was split into two, using an optical coupler. On the signal branch, the light is externally modulated around the operating frequency $v_{R F}$ (set at $6 \mathrm{GHz}$ ) using an EOM driven by the vector network analyzer (VNA) RF output. The lower frequency sideband was filtered out by using a fiber Bragg grating (FBG) notch filter. Hereafter, the higher sideband is called RF subcarrier. So, the optical spectrum at the output of the notch filter contains two monochromatic lines, at the carrier frequency $v_{c}$, and at a frequency around $v_{c}+6 \mathrm{GHz}$. Then this single sideband modulated signal was delivered into the DSF.

The other branch was also split for the generation of two separate Brillouin pumps at different frequencies. The first branch was modulated at $v_{B}+v_{\mathrm{RF}}$ using an EOM driven by an RF synthesizer, and the carrier was completely suppressed by applying an adequate bias to the EOM. Only the higher frequency sideband was selected using a FBG to be used as Pump 1 and then strongly boosted using an erbium doped fiber amplifier (EDFA) to generate strong dispersion around RF subcarrier, thus producing sufficient group delays for microwave signals. The optical power of Pump 1 was controlled using a variable optical attenuator to precisely adjust the amount of group delay. The optical phase of Pump1 was externally modulated by a noise generator through an external electro-optic phase modulator in order to broaden its spectrum. So, the spectral width of Pump 1 in the output of the modulator was simply tuned by varying the amplitude of the white noise applied to the phase modulator. The maximal bandwidth we obtained in this experiment was $120 \mathrm{MHz}$.

The light from the second branch was also modulated through an EOM, at the RF frequency $v_{B}+\delta v$, and the carrier was fully suppressed following the same scheme. The higher and lower frequency sidebands led to Brillouin gain and loss resonances, respectively, shifted by $+\delta v$ and $-\delta v$ with respect to the carrier frequency, with identical depth and spectral shape. Therefore, the amplitude of the carrier remains constant since the gain and loss for the carrier mutually cancel out, while the associated optical phase shift imparted on the carrier is doubled [8]. The detuning of the resonance frequency $\delta v$ was then accurately adjusted within a $15 \mathrm{MHz}$ span, so as to induce the proper phase shift to obtain TTD corresponding to the dispersion slope induced by Pump 1 and applied to the RF signal. 

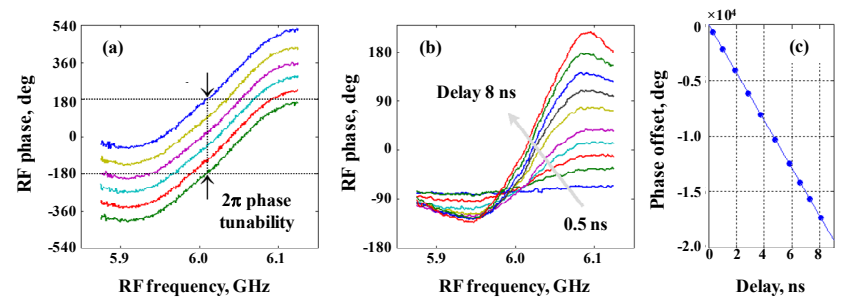

Figure 3: (a) Fixed RF signal delay, and tuning of the optical carrier phase shift: $2 \pi$ phase tuneability is achieved. (b) Fixed optical carrier phase shift and tuning of the RF signal delay from 0.5 to $10 \mathrm{~ns}$ and (c) associated phase offset as a function of the delay.

\section{TRUE TIME DELAY}

Figure 3(a) first shows the phase of the detected RF signal as a function of the RF frequency, when the optical carrier phase shift is tuned using the SBS-based phase shifter. The pump power of the SBS delay line is fixed and corresponds to a $10 \mathrm{~ns}$ delay. $2 \pi$ phase tunability is obtained, which was mandatory to compensate any value of $\varphi_{\text {offset }}$. Figure 3(b) shows a similar measurement when this time the pump power of the SBS delay line is varied, while the optical carrier phase shift is fixed. The SBS gain spectrum is centered at $6.02 \mathrm{GHz}$ from the optical carrier. For each pump power, the measured phase shift exhibits a linear variation with respect to the RF frequency from $5.955 \mathrm{GHz}$ to $6.075 \mathrm{GHz}$, showing a bandwidth of $120 \mathrm{MHz}$. Within this bandwidth, the delay varies from 0.5 to $8 \mathrm{~ns}$ when the SBS pump power is increased. Figure 3(c) then shows the phase offset as a function of the applied delay corresponding to the data shown in Figure 3(b). For true-time delay operation, the optical carrier phase shift has then to be adjusted for each delay in order to eventually obtain a constant phase offset with respect to the delay.

Figure 4 shows the experimental validation of this concept for delays of $0.03,1.5,4.8,7.5$ and $9.9 \mathrm{~ns}$. On the central figure, the unwrapped RF phase is plotted versus the RF frequency, and the linear regions of the phase curves have been extrapolated down to the origin of frequencies. The right insets show zooms of the phase curves within the Brillouin
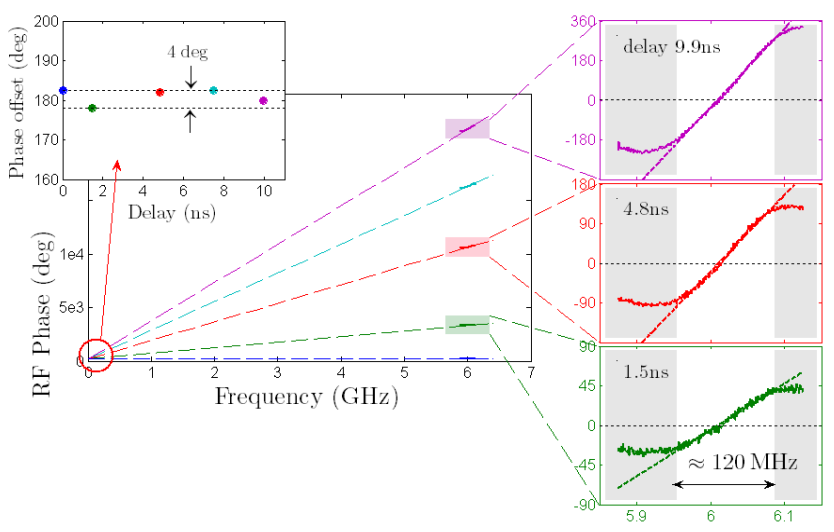

Figure 4: SBS-SCT based true-time delay line. For delays from 0.03 to $9.9 \mathrm{~ns}$, the optical carrier phase shift is adjusted to ensure a constant extrapolated phase offset at the origin of RF frequencies (top inset). A $120 \mathrm{MHz}$ instantaneous bandwidth is obtained. spectral bandwidth for delays of 1.5, 4.8 and 9.9 ns. On the top inset, we plotted the residual phase offset after compensation by the SBS-based phase shifter. For all delays, the phase offset is equal to $180^{\circ} \pm 2^{\circ}$. The SCT delay line therefore operates as a TTD element, providing a tuneable TTD up to $10 \mathrm{ns,} \mathrm{within}$ an instantaneous RF bandwidth of $120 \mathrm{MHz}$. The operating frequency of $6 \mathrm{GHz}$ was in our case limited by equipment availability, but the SBS-based SCT technique can be in principle applied at any operating frequency.

\section{DYNAMIC MICROWAVE PHOTONIC FILTER}

We implemented a two-tap based notch-type incoherent microwave photonic filter (MPF), as shown in Figure 5. A $7.5 \mathrm{~m}$-long optical fiber was inserted in a branch of the MPF to provide the target frequency response. The imbalance in optical path length between the two branches offers the possibility to study the feasibility of the SCT technique by investigating several resonances of the MWP filter. Finally, the outputs from the two branches of the MPF are combined in the output coupler and the received RF signal is compared to the input to obtain the filter RF transfer function with a VNA.

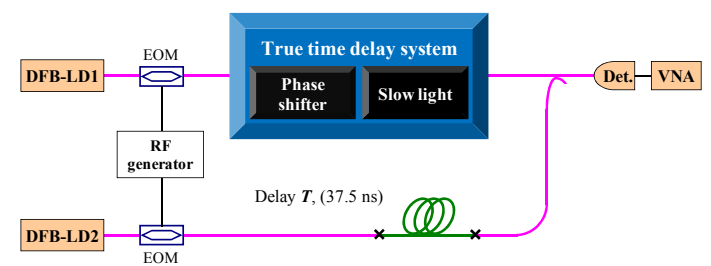

Figure 5: Experimental layout of dynamically reconfigurable microwave photonic filter, based on stimulated Brillouin scattering in fibers.

To check the filter tunability, we first measured the effect of a pure phase shift on the optical carrier by recording the filter spectral transfer function. In this case it corresponds to a complex-valued two-coefficient MPF $[1,9,10]$. Figure 6 displays the frequency response of the filter obtained with the SBS pump turned on and thus a fixed TTD, preserving the free spectral range. In this figure, continuous tunability was demonstrated as the phase of the lower-coefficient tap is modified by tuning the carrier phase shift using SBS-based phase shifter. Experimental measurements (symbols) are

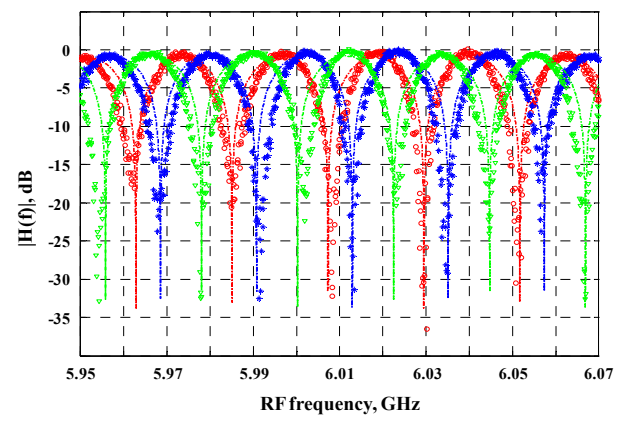

Figure 6: Measured (symbol) and simulated (dashed line) filter frequency response (a) tuning the carrier phase shift for $110 \mathrm{~mW}$ Brillouin pump power. 
compared to theoretical calculations (dashed lines) showing excellent agreement. The observed small deviations of the RF response from the ideal behavior are attributed to the noncompletely flat phase response generated by SBS system. It must be considered that a noise source is used to broaden the Brillouin gain, resulting in small deviations from the Gaussian gain shape and thus in phase fluctuations.

It must be pointed out that the central frequency of the notch filter is varied while maintaining the shape of the transfer function unaltered, since the true time delay between the arms of the MPF is fixed. The maximum achievable phase shift is greater than $360^{\circ}$, fact which leads to a continuously full tuning range of the filter response. This technique can be implemented to obtain a RF frequency-independent phase shift; in our case the filter central frequency is practically limited by our instrumentation.

To get the tunability in the Free Spectral Range, we implemented a SBS-based tunable delay in one of the branch. The two branches of the MPF have a path imbalance of $7.5 \mathrm{~m}$, corresponding to $37.5 \mathrm{~ns}$ and resulting in a FRS of $26.7 \mathrm{MHz}$. TTD can be generated using the SCT technique, which should result in a different spectral response of the MPF, since its FSR will change upon tuning the delay. Figure 7 confirms that the FSR of the filter changes when the pump is applied. When a $110 \mathrm{~mW}$ pump is launched into the MPF lower branch, SBS generates a TTD of about $7 \mathrm{~ns}$ at $6 \mathrm{GHz}$ over a $120 \mathrm{MHz}$ bandwidth. This means that the FSR will be decreased down to $22.5 \mathrm{MHz}$, which corresponds to a $20 \%$ FSR reduction. A SBS system is used as well for the carrier phase adjustment, as required by the SCT technique [4]. The phase of the microwave signal can be expressed as:

$$
\phi\left(\omega_{R F}\right)=\phi\left(\omega_{c}\right)-\phi\left(\omega_{s}\right)+\left.\omega_{R F} \frac{\partial \phi\left(\omega_{c}\right)}{\partial \omega}\right|_{\omega_{c}-\omega_{s}},
$$

where $\phi\left(\omega_{\mathrm{c}}\right)$ and $\phi\left(\omega_{\mathrm{s}}\right)$ are the phase shifts at the carrier and sideband frequency respectively. To achieve TTD, $302.4^{\circ}$ of carrier compensation is required in our particular case illustrated in Figure 7. The SBS-based phase shifter allows us to fix the origin of the FSR variation. A notch filter with more than $30 \mathrm{~dB}$ extinction ratio has been so achieved.

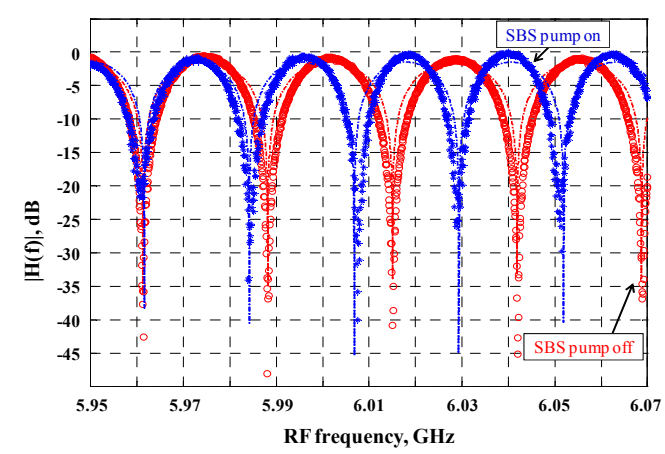

Figure 7: Measured (symbol) and simulated (dashed line) filter frequency for $110 \mathrm{~mW}$ Brillouin pump power and for no pump.

\section{CONCLUSION}

We have experimentally demonstrated that Brillouin slow light elements offer novel and outstanding capabilities in microwave photonics systems, particularly thanks to an innovative all-optical tuneable true-time delay line based on the separate carrier tuning technique. We implemented two independently controlled SBS systems to individually tune the optical carrier phase and the RF signal delay. As a result, a TTD continuously tuneable from 0 to $10 \mathrm{~ns}$ was achieved, within an instantaneous bandwidth of $120 \mathrm{MHz}$, and at an operating frequency of $6 \mathrm{GHz}$. However, it must be pointed out that this technique has no physical limitation on the operating RF frequency and signal bandwidth since the Brillouin resonance can be positioned at any desired RF frequency by shifting the pump frequency. Brillouin systems offer a high flexibility to operate at bandwidth up to a few $\mathrm{GHz}$. By extrapolating the performances of this setup used in a potential phased array antennas feeding system, we showed that the SCT technique meets the requirements for $\pm 45^{\circ}$ scanning at any operating frequency and with an instantaneous bandwidth of $8 \%$. Besides, we have experimentally demonstrated the implementation of the SBS-based SCT technique in a reconfigurable microwave photonic notch filter operating around $6 \mathrm{GHz}$. The tunable TTD induced in our setup allowed a $20 \%$ tunability of FSR of the filter, and the optical phase compensation on the carrier then enabled to control the origin of the FSR variation, matching the reference frequency. Therefore, we believe that this technique can be an unmatched and highly promising solution for microwave photonics applications.

\section{REFERENCES}

[1] J. Capmany and D. Novak, "Microwave photonics combines two worlds," Nature Photonics, 1, 319-330 (2007).

[2] R. W. Boyd and D.J. Gauthier, "'Slow' and 'Fast' light," Ch. 6 in Progress in Optics 43, E. Wolf, Ed. (Elsevier, Amsterdam, 2002), 497530 .

[3] J. B. Khurgin and R. S. Tucker, Slow light: Science and applications (CRC Press, Boca Raton, 2009).

[4] P. A. Morton and J. B. Khurgin, "Microwave Photonic Delay Line With Separate Tuning of the Optical Carrier," Photon. Technol. Lett., 21, 1686-1688, (2009).

[5] Luc Thévenaz, "Slow and fast light in optical fibers," Nature Photonics, 2, 474-481 (2008).

[6] J. Sancho, S. Chin, M. Sagues, A. Loayssa, J. Lloret, I. Gausulla, S Sles, L. Thevenaz and J. Capmany, "Dynamic Microwave Photonic Filter using Separate Carrier Tuning based on Stimulated Brillouin scattering in Fibers," Photon. Technol. Lett., in press (2010).

[7] M. Nikles, L. Thévenaz, P. A. Robert, "Brillouin gain spectrum characterization in single mode optical fibers," J. Lightwave Technol., 15, 1842-1851 (1997).

[8] Al. Loaysa and F. J. Lahoz, "Broad-band RF photonic phase shifter based on stimulated Brillouin scattering and single-sideband modulation," Photon. Technol. Lett., 18, 208-210 (2006).

[9] W. Xue, S. Sales, J. Mork and J. Capmany, "Widely Tunable Microwave Photonic Notch Filter Based on Slow and Fast Light Effects," Photon. Technol. Lett. 21, 167-169 (2009).

[10] M. Sagues, R. Garcia, A. Loayssa, S. Sales and J. Capmany, "Multi-tap complex-coefficient incoherent microwave photonic filters based on optical single-sideband modulation and narrow band optical filtering," Opt. Express, 13, 295-303 (2008). 